\title{
Research on the 3D construction of plant morphology
}

\author{
Zhiyong $\mathrm{He}^{\mathrm{a}}$, Xiaopeng Dai ${ }^{\mathrm{b}}$ \\ School of information Science \& Technology of Hunan Agricultural University ChangSha China \\ ahe_zhiyong@sina.comb daixpedu@126.com
}

Keywords: Hough transform;voting;3D reconstruction.

Abstract. In this paper, according to the principle of Hough transform, using the multi - directional image information of an object to calculate the pixel points of the object in the space, and get the real three-dimensional image from the object surface texture mapping which is composed of pixel points. This method is good for the reconstruction of plant root, branches and flowers. There's a certain novelty value in this approach, such as the simple algorithm, simple operation, good robustness, and can be implemented on the PC to achieve the level of full automation and real-time processing, so it has important application prospect.

\section{Introduction}

It is a very difficult and attractive project in computer vision to reconstruct three-dimensional images of the original space objects based on the projection of the object obtained by different viewpoints. ${ }^{[1]}$ Compared with the Facial reconstruction and building reconstruction, the common point is that the structure of the reconstructed object is simple, and the volume is large, so it is not necessary to consider the problem of occlusion, and the precision is not too high. But with the development of information science, 3D reconstruction problem of some other objects will also have more and more important significance, such as plant roots and stems of $3 \mathrm{D}$ reconstruction is an important tool for plant growth modeling and plant nutrition analysis. These objects may be smaller but more occluded, and the reconstruction accuracy is high, so it is more difficult. In this paper, based on the characteristics of the root system and the branch rod structure, a reconstruction algorithm based on Hough transform is proposed. This method is simple and easy, so it is fast, Also not too high requirements for camera calibration precision, and it can overcome the problem of occlusion and incomplete information.

\section{Basic principle}

Hough in the detection of binary of image in simple geometries proposed a method, this method was later called Hough transform ${ }^{[2]}$, it is widely applied in the image. In this paper, the principle is applied to 3D reconstruction. We first simply introduce the Hough transform of a straight line,in order to drive it home.

We know that the parameter equation of the straight line is: $y=a x+b$,so in parameter space $(a, b)$,every dot $\left(a^{\prime}, b^{\prime}\right)$ represents a straight line. Assuming in the image plane $(x, y)$, there is a straight line through $\left(x_{1}, y_{1}\right),\left(x_{2}, y_{2}\right),\left(x_{3}, y_{3}\right)$, 


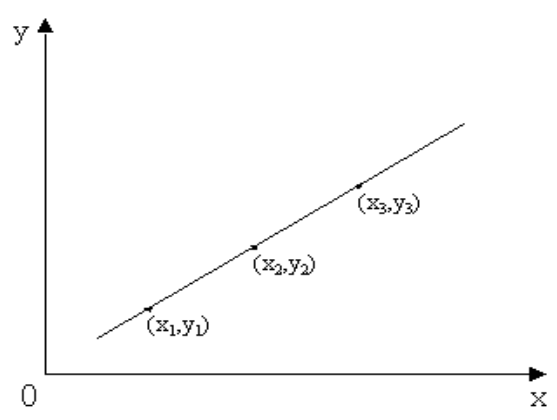

Figla Straight line on the image plane

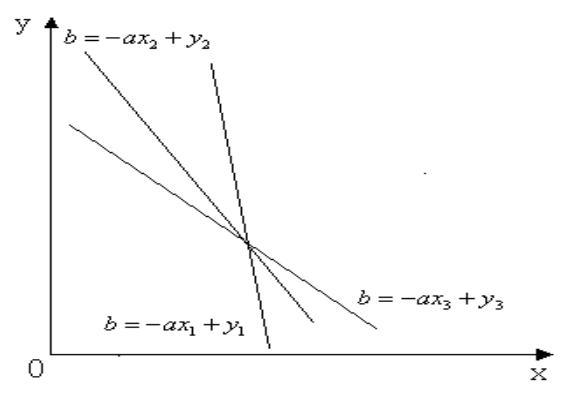

Fig1b Representation of straight line in parameter space

(As fig 1a), In parameter space $(a, b), b=-a x_{1}+y_{1}, b=-a x_{2}+y_{2}, b=-a x_{3}+y_{3}$ will cross at a point (As fig 1b).

\section{The experimental steps and results}

Synthesized the 3D entity image of the object by Hough transform. The specific method of voting is as follows:

Assume $^{\left(x^{(1)}, y^{(1)}\right)}$ is the first of the value of the image of binarization after 1 points (that is, black spot),we select the first frame image projection direction as the starting direction(is show in fig2a), then it could be from $(X, Y, Z)$

$$
\begin{aligned}
& X=x^{(1)}\left(Z_{0}+i\right) / f_{x} \\
& Y=y^{(1)}\left(Z_{0}+i\right) / f_{y} \\
& Z=Z_{0}+i
\end{aligned}
$$
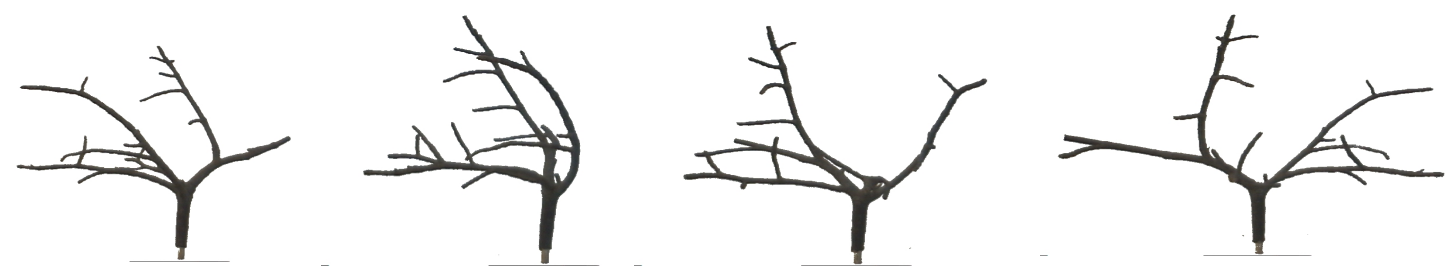

Fig 2a Original image 

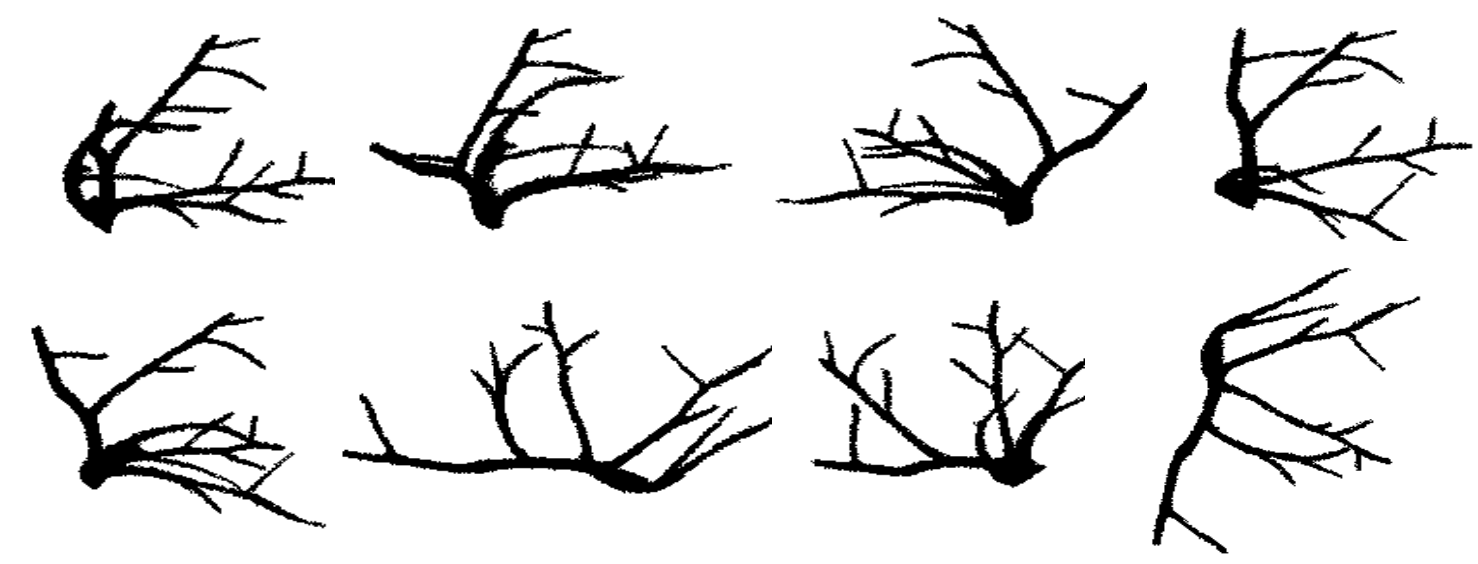

Fig $2 b$ Projection of the reconstructed image

The specific method of voting is as show in fig $2 b$,

Assume $^{\left(x^{(1)}, y^{(1)}\right)}$ is the first of the value of the image of binarization after 1 points (that is, black spot),we select the first frame image projection direction as the starting direction,then it could be from $(X, Y, Z)[3]$

$$
\begin{aligned}
& X=x^{(1)}\left(Z_{0}+i\right) / f_{x} \\
& Y=y^{(1)}\left(Z_{0}+i\right) / f_{y} \\
& Z=Z_{0}+i \\
& i=0, \pm 1, \pm 2, \cdots, \pm M
\end{aligned}
$$

$M$ is the location of the object distribution, ${ }^{Z_{0}}$ Is the rotary shaft to the position of the camera optical center,so, We cast a vote on all such points $(X, Y, Z)$. the rest may be deduced by analogy,assume the $\mathrm{n}$ frames in the image $\left(x^{(n)}, y^{(n)}\right)$ is a black spoot,and from frame 1 to frame the objects and turntable turn $\theta_{n}$ corners in a counter clockwise direction, we cast a vote on the following $(X, Y, Z)$, among them ${ }^{[4]}$,

$$
\begin{aligned}
& X=\left\lfloor x^{(n)}\left(Z_{0}+i\right) / f_{x}\right\rfloor \cos \theta_{n}+\left(Z_{n}+i-Z_{0}\right) \sin \theta_{n} \\
& Y=y^{(n)}\left(Z_{0}+i\right) / f_{y} \\
& Z=Z_{0}-\left[x^{(n)}\left(Z_{0}+i\right) / f_{x}-Z_{0}\right] \sin \theta_{n}+\left(Z_{0}+i-Z_{0}\right) \cos \theta_{n} \\
& i=0, \pm 1, \pm 2, \cdots, \pm M
\end{aligned}
$$

When all of the selected images and all the pixels are scanned, calculating the votes, We stipulate that object space of arbitrary point $(X, Y, Z)$ in each frame of the image to vote at least 
have one vote, so we can choose $\mathrm{N}$ to vote total frames as a benchmark to determine threshold $\mathrm{T}$, generally we choose ${ }^{[5]}$ (as in show fig3)
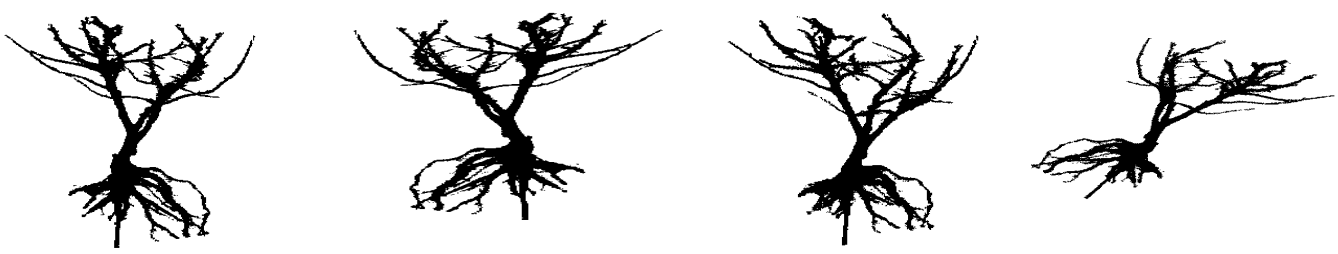

Fig3 Reconstructed image of tree

$T=80 \% N$.Fig5 chose $N=36, T=90 \% N \approx 32$. Of course, it is not appropriate to put objects and only one camera to obtain information, and sometimes it is easy to lose some information, especially the top of the information, so as to affect the effect of the reconstruction ${ }^{[6]}$. In order to overcome this difficulty, we can use the lifting camera, changing the direction of the object, and the dual camera to obtain images and other ways to vote, The 3D image model shown in Figure 3 with the number of votes greater than threshold set point that is composed of objects. To check and display the results, chose proper proportion and use the mouse to flip the object design program, to watch all directions projection information.

For some actual need, we can color texture mapping to the surface of the object. Fig. 4 is the color value of the projection on the surface of the flower, the results is that the color is darker than the actual, but the texture is still clear, if use paste method, the effect will be better, here is not a detailed discussion.

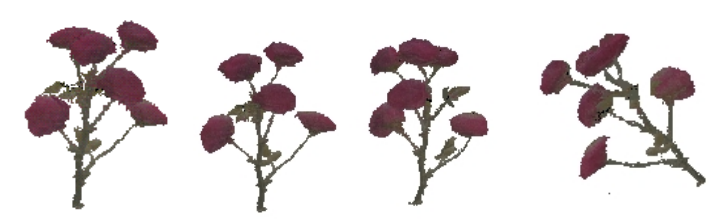

Fig 4 Reconstruction object of texture mapping

\section{Discussion}

The above experimental accuracy at least can reach1/10 millimeter, if use of camera microscope or precision higher turntable and more accurate calibration, the precision can also further improve. From the above experimental results, it can be seen that the effect of this method is satisfactory, especially for the construction of plant roots, rods and sticks, However, this method is constructed in the image is a three-dimensional entity, need large memory space for the reconstruction of large objects, is unable to withstand for the current market PC, so it need improve. Hope one day can generate on the surface of the object image, or to make the 3D mesh model, which can be edited, and can be applied to some other important areas of face modeling and face recognition, which needs to be further studied in the future. 


\section{Acknowledgments.}

Project supported by the Research of Education Bureau of Hunan Province,China(Grant No.12C0154).

\section{Reference}

[1] Ma Songde,Zhang Zhengyou, Computer Vision-CComputational Theory and Algorithm, science press, $1998,50-110$.

[2] P.V.C. Hough, Method and Means for Recognizing Complex Patterns. U.S. Patent 3069654, 1962.

[3] Kutulakos K. N., Seitz S.M., A Theory of Shape by Space Carving. Computer Vision, 1999, The Proceedings of the Seventh IEEE International Conference On,Volume:1,1999.

[4] Cui Yi,Digital Image Processing Technology and Application,Electronic Press,P100 107,1996 。

[5] Liao Hong, Ge Zhenyang, Yan Xiaolong. Simulation and Application of Phosphorus Uptake by Plants under Water and Phosphorus Coupling Stress,Chinese Sciences Bulletin,2001,46 ( 8 ) 641 $-646$.

[6] Schmitt F., Yemez Y., 3D Color Object Reconstruction From 2D Image Sequences. Image Processing, 1999, ICIP99, Proceeding International Conference On, Volume:1999,p65-69 . 